\title{
cDNA cloning of a novel human gene NAKAP95, neighbor of A-kinase anchoring protein 95 (AKAP95) on chromosome 19p13.11-p13.12 region
}

Received: July 13, 1999 / Accepted: September 4, 1999

\begin{abstract}
A-kinase anchoring protein 95 (AKAP95) is a nuclear protein which binds to the regulatory subunit (RII) of cyclic adenosine monophosphate (cAMP)-dependent protein kinase (PKA) and to DNA. A novel nuclear human gene which shares sequence homology with the human $A K A P 95$ gene was identified by a nuclear transportation trap method. By polymerase chain reaction (PCR)-based analysis with both a human/rodent monochromosomal hybrid cell panel and a radiation hybrid panel, the gene was mapped to the chromosome 19p13.11-p13.12 region between markers WI-4669 and CHLC.GATA27C12. Furthermore, alignment with genomic sequences revealed that the gene and human AKAP95 resided tandemly only approximately $250 \mathrm{bp}$ apart from each other. We designated this gene as neighbor of AKAP95 (NAKAP95). The exonintron structure of NAKAP95 and AKAP95 was conserved, indicating that they may have evolved by gene duplication. The predicted protein product of the NAKAP95 gene consists of 646 amino acid residues, and NAKAP95 and AKAP95 had an overall $40 \%$ similarity, both having a potential nuclear localizing signal and two $\mathrm{C} 2 \mathrm{H} 2$ type zinc finger motifs. The putative RII binding motif in AKAP95 was not conserved in NAKAP95. A reverse transcription coupled (RT)-PCR experiment revealed that the NAKAP95 gene was transcribed ubiquitously in various human tissues.
\end{abstract}

N. Seki · T. Saito

Genome Research Group, National Institute Radiological Sciences, Chiba, Japan

N. Seki

Laboratory of Gene Function II, Kazusa DNA Research Institute, Chiba, Japan

N. Ueki $\cdot$ K. Yano $\cdot$ Y. Masuho $\cdot$ M. Muramatsu $(\bowtie)$

Helix Research Institute, 1532-3 Yana, Kisarazu, Chiba 292-0812, Japan

Tel. +81-438-52-3966; Fax +81-438-52-3952

e-mail: mmasaaki@hri.co.jp

M. Muramatsu

Department of Biological Cybernetics, Medical Research Institute,

Tokyo Medical Dental University, Tokyo, Japan
Key words Cyclic AMP-dependent protein kinase (PKA) · A-kinase anchoring proteins (AKAPs) $\cdot$ AKAP95 $\cdot$ Chromosome 19p13.11-p13.12 - RH mapping · Genomic structure $\cdot$ Gene duplication

\section{Introduction}

A large number of hormones and neurotransmitters utilize cyclic adenosine monophosphate (cAMP) as an intracellular second messenger. Cyclic AMP regulates a number of key cellular processes such as metabolism, gene regulation, cell growth, cell differentiation, ion channel conductivity, and release of synaptic vesicles (Krebs and Beavo, 1979; Boynton and Whitfield, 1983; Edelman et al. 1987; Roesler et al. 1988; Taylor et al. 1990; McKnight 1991). The main intracellular target for cAMP in mammalian cells is cAMPdependent protein kinase (PKA or A-kinase). PKA type II is directed to different subcellular loci through interaction of the RII subunits with A-kinase anchoring proteins (AKAPs) (Scott and Macartney, 1994; Rubin 1994; Hausken et al. 1996; Hausken and Scotte, 1996; Faux and Scott 1996). A number of different AKAPs which direct different compartmentalizations have been found: AKAP79/75 direct the RII to postsynaptic densities and cortical actin (Carr et al. 1992; Li et al. 1996), AKAP250/ Gravin to filopodia (Nauert et al. 1997), AKAP350 to centrosomes (Schmidt et al. 1999), AKAP100 to sarcoplasmic reticulum (McCartney et al. 1995), AKAP220 to peroxisome (Lester et al. 1996), AKAP85 to Golgi apparatus (Keryer et al. 1993), and AKAP84/149 to mitochondria (Chen et al. 1997).

AKAP95 was originally isolated by an interaction cloning strategy with RII $\alpha$ as a probe from a rat pituitary $\left(\mathrm{GH}_{4} \mathrm{C}_{1}\right)$ cDNA library (Coghlan et al. 1994). The rat AKAP95 contained both RII and DNA binding domains. The AKAP95 was detected in a nuclear matrix fraction, and immunofluorescence, using purified anti-AKAP95 antibodies, revealed distinct nuclear staining in a variety of cell types (Coghlan et al. 1994). It is proposed that AKAP95 
could play a role in targeting type II PKA for cAMPresponsive nuclear events. Recently, human AKAP95 was identified and sequenced, and was shown to be $89 \%$ homologous to rat AKAP95 (Eide et al. 1998).

We have recently developed a screening method, designated nuclear transportation trap (NTT), to systematically isolate nuclear proteins (Ueki et al. 1998). Using this method, several novel nuclear genes were isolated, such as CLIM1/CLIM2, PIAS3, and HFB30 (Ueki et al. 1999a,b,c). We have also isolated a partial cDNA clone which had a sequence homologous to the human $A K A P 95$ gene. We describe here the complete cDNA sequence, expression profile, chromosomal assignment, and genomic structure of the gene, NAKAP95.

\section{Cloning of human NAKAP95 gene}

A partial cDNA clone (initially called HFB2018) was isolated from a human fetal library, using the NTT method (Ueki et al. 1998). BLAST search revealed that HFB2018 was most homologous to human $A K A P 95$ (Eide et al. 1998). Specific primers were designed, according to the HFB2018 sequence, to obtain a full-length cDNA from a human fetal brain library, using GeneTrapper (GIBCO BRL, Gaithersburg, MD, USA). The resultant cDNA was $2057 \mathrm{bp}$ in length and contained an open reading frame of 646 amino acids (Fig. 1a). The nucleotide sequence of the cDNA will appear in GenBank/EMBL/DDBJ databases under the accession number, AB025905. Since the gene was found to reside next to the human AKAP95 gene (see below and Fig. 2a) we designated this clone as neighbor of $A K A P 95$ (NAKAP95). The predicted NAKAP protein initiated from the first putative initiation ATG codon was in accordance with Kozak's rule (Kozak 1987). A canonical polyadenylation signal, AATAAA, was located $14 \mathrm{bp}$ upstream of a poly(A) (Fig. 1a). The alignment of predicted amino acid sequences of human AKAP95, rat AKAP95, and human NAKAP95 proteins is shown in Fig. 1b. Human NAKAP95 and AKAP95 are 30\% identical (40\% similar) at the amino acid level. NAKAP95 and AKAP95 possess two $\mathrm{C} 2 \mathrm{H} 2$ type zinc finger motifs at a similar position. The regions that included the zinc finger motifs showed the highest identity between the two proteins. A putative nuclear localization signal KKKKRK was found at residues 274-279 (Fig. 1a). From the alignment, the putative RII-binding region of AKAP95 (Coghlan et al. 1994; Hausken and Scott 1996) was not conserved in NAKAP95. Therefore, whether NAKAP95 can bind to RII remains elusive.

\section{Expression profile of human NAKAP95 gene}

We examined the distribution of the $N A K A P 95$ transcript in various human tissues by reverse transcription-coupled polymerase chain reaction (RT-PCR) as described previously (Seki et al. 1998; 1999). Primers used for the RT-PCR corresponded to the coding region of the gene (5'-TGG TGC CGC ATT TGG AGA CAG-3') and (5'-TGC CAA ACC CGA AAC CAA AGC-3'). The primer set gave a longer PCR product from genomic DNA, which was easily distinguished from the 459-bp product from the mRNA. The 459-bp PCR product was generated in all tissues examined, indicating that the transcript is ubiquitously expressed in a wide variety of human tissues (Fig. 3). Therefore, NAKAP95 described in the present study seems to be involved in a basic house-keeping function of cells.

\section{Chromosome mapping and genomic structure of human NAKAP95 gene}

Chromosomal assignment of the human NAKAP95 gene was done by PCR analysis of a human/rodent somatic cell hybrid panel (National Institute of General Medicine Service, Coriell Cell Repositories, Camden, NJ, USA) and a radiation hybrid panel (Genebridge 4; Research Genetics, Huntsville, AL, USA), as described previously (Saito et al. 1997; Seki et al. 1997). The human NAKAP95 specific PCR primers (5'-TCG GCT GCC CTC CCT CTT CTC-3', 5'GGT CCG CCT CAT CTG CTT CAT-3') gave rise to an amplified product with a size of $139 \mathrm{bp}$ by genomic PCR. First, the specific amplified product for human was detected only from the hybrid containing human chromosome 19 (data not shown). Further mapping analysis, using a radiation hybrid panel with the same primer set, was done. Statistical analysis of the radiation hybrid data was performed using the RHMAPPER software package (http:// carbon.wi.mit.edu:8000/cgi-bin/contig/rhmapper.pl). The data vector for the human NAKAP95 gene was 0000000101 $0001000010 \quad 1000111110 \quad 0001010000 \quad 1100010101$ 1011010001000100011011010110010001011100001 and the consequent report indicated that the gene was placed to $3.89 \mathrm{cR}$ distal from the marker CHLC.GATA27C12 (lod > $3.0)$. The region including the marker was cytogenetically mapped to the 19p13.11-p13.12 region (Fig. 2a). Since human NAKAP95 and AKAP95 genes have been mapped to the same region, we then searched for genomic sequences in the public database, and found a cosmid clone which contains both genes (accession number, AC005785). The human NAKAP95 and AKAP95 resided tandemly and the first exon of human $A K A P 95$ began $258 \mathrm{bp}$ after the end of the NAKAP95 gene. We have no data about the orientation of NAKAP95 and AKAP95 in chromosome 19. To confirm that human $A K A P 95$ and NAKAP95 are juxtaposed, primers were designed between the last exon of human $N A K A P 95$ and the first exon of $A K A P 95$ (5'-GAC AGC CCC GAG GAG GAG AAG-3' and 5'-CCC ACC AGC AGC CCC GTT TAC-3'; product size, 668bp). Genomic PCR using the primers generated a PCR product of the expected size (data not shown), proving that human AKAP95 and NAKAP95 are next to each other. Although the sequence between the genes would provide the promoter for the $A K A P 95$ gene, it lacked a typical TATA box or a $\mathrm{GC}$-rich region. 
Fig. 1. a Nucleotide sequence and deduced amino acid sequence of human NAKAP95 gene. Asterisk denotes the stop codon. Two $\mathrm{C} 2 \mathrm{H} 2$ zinc finger motifs are underlined and a putative nuclear localizing signal is shown in italics. The polyadenylation signal, aataaa is double underlined. The nucleotide sequence of the NAKAP95 gene is deposited in GenBank/EMBL/DDBJ databases under the accession number, AB025905. The nucleotide sequences of both strands were determined by a primer walking method, using an ABI377 sequencer (Perkin Elmer, Norwalk, CT, USA). b Alignment of human AKAP95 (accession number, Y11997), rat AKAP95 (accession number, U01914), and human NAKAP95 (accession number, AB025905) proteins. Identities are indicated by black background and similar residues are shadowed. Asterisks denote the termination codon
gagcagcagaagccggcgtcgtcggatgttgtgttgcccgccaccATGAGCTACACAGGC

$$
\begin{array}{lllll}
M & S & Y & T & G
\end{array}
$$

TTTGTCCAGGGATCTGAAACCACTTTGCAGTCGACATACTCGGATACCAGCGCTCAGCCC $\begin{array}{llllllllllllllllllll}F & V & Q & G & S & E & T & T & L & Q & S & T & Y & S & D & T & S & A & Q & P\end{array}$ ACCTGTGATTATGGATATGGAACTTGGAACTCTGGGACAAATAGAGGCTACGAGGGCTAT $\begin{array}{lllllllllllllllllllll}T & C & D & Y & G & Y & G & T & W & N & S & G & T & N & R & G & Y & E & G & Y\end{array}$ GGCTATGGCTATGGCTATGGCCAGGATAACACCACCAACTATGGGTATGGTATGGCCACT $\begin{array}{lllllllllllllllllllllll}G & Y & G & Y & G & Y & G & Q & D & N & T & T & N & Y & G & Y & G & M & A & T\end{array}$ TCACACTCTTGGGAAATGCCTAGCTCTGACACAAATGCAAACACTAGTGCCTCGGGTAGC $\begin{array}{llllllllllllllllllll}S & H & S & W & E & M & P & S & S & D & T & N & A & N & T & S & A & S & G & S\end{array}$ GCCAGTGCCGATTCCGTTTTATCCAGAATTAACCAGCGCTTAGATATGGTGCCGCATTTG $\begin{array}{lllllllllllllllllllll}A & S & A & D & S & V & L & S & R & I & N & Q & R & L & D & M & V & P & H & L\end{array}$ GAGACAGACATGATGCAAGGAGGCGTGTACGGCTCAGGTGGAGAAAGGTATGACTCTTAT $\begin{array}{lllllllllllllllllllllllll}E & T & D & M & M & Q & G & G & V & Y & G & S & G & G & E & R & Y & D & S & Y\end{array}$ GAGTCCTGCGACTCGAGGGCCGTCCTGAGTGAGCGCGACCTGTACCGGTCAGGCTATGAC $\begin{array}{llllllllllllllllllll}E & S & C & D & S & R & A & V & L & S & E & R & D & L & Y & R & S & G & Y & D\end{array}$ TACAGCGAGCTTGACCCTGAGATGGAAATGGCCTATGAGGGCCAATACGATGCCTACCGC $\begin{array}{llllllllllllllllllll}Y & S & E & L & D & P & E & M & E & M & A & Y & E & G & Q & Y & D & A & Y & R\end{array}$ GACCAGTTCCGCATGCGTGGCAACGACACCTTCGGTCCCAGGGCACAGGGCTGGGCCCGG $\begin{array}{llllllllllllllllllllll}D & Q & F & R & M & R & G & N & D & T & F & G & P & R & A & Q & G & W & A & R\end{array}$ GATGCCCGGAGCGGCCGGCCAATGGCCTCAGGCTATGGGCGCATGTGGGAAGACCCCATG $\begin{array}{lllllllllllllllllllll}D & A & R & S & G & R & P & M & A & S & G & Y & G & R & M & W & E & D & P & M\end{array}$ GGGGCCCGGGGCCAGTGCATGTCTGGTGCCTCTCGGCTGCCCTCCCTCTTCTCCCAGAAC

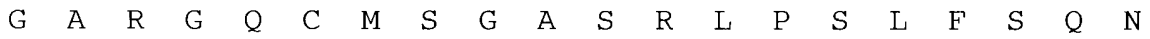
ATCATCCCCGAGTACGGCATGTTCCAGGGCATGCGAGGTGGGGGCGCCTTCCCGGGCGGC $\begin{array}{llllllllllllllllllllll}I & I & P & E & Y & G & M & F & Q & G & M & R & G & G & G & A & F & P & G & G\end{array}$ TCCCGCTTTGGTTTCGGGTTTGGCAATGGCATGAAGCAGATGAGGCGGACCTGGAAGACC $\begin{array}{llllllllllllllllllllllllll}S & R & F & G & F & G & F & G & N & G & M & K & Q & M & R & R & T & W & K & T\end{array}$ TGGACCACAGCCGACTTCCGAACCAAGAAGAAGAAGAGAAAGCAGGGCGGCAGTCCTGAT $\begin{array}{llllllllllllllllllll}\mathrm{W} & \mathrm{T} & \mathrm{T} & \mathrm{A} & \mathrm{D} & \mathrm{F} & \mathrm{R} & \mathrm{T} & K & K & K & K & R & K & \mathrm{Q} & \mathrm{G} & \mathrm{G} & \mathrm{S} & \mathrm{P} & \mathrm{D}\end{array}$ GAGCCAGATAGCAAAGCCACCCGCACGGACTGCTCGGACAACAGCGACTCAGACAATGAT $\begin{array}{llllllllllllllllllll}E & \mathrm{P} & \mathrm{D} & \mathrm{S} & \mathrm{K} & \mathrm{A} & \mathrm{T} & \mathrm{R} & \mathrm{T} & \mathrm{D} & \mathrm{C} & \mathrm{S} & \mathrm{D} & \mathrm{N} & \mathrm{S} & \mathrm{D} & \mathrm{S} & \mathrm{D} & \mathrm{N} & \mathrm{D}\end{array}$ GAGGGCACCGAGGGGGAAGCCACAGAGGGCCTTGAAGGCACCGAGGCTGTGGAGAAGGGC $\begin{array}{llllllllllllllllllll}E & G & T & E & G & E & A & T & E & G & L & E & G & T & E & A & V & E & K & G\end{array}$ TCCAGAGTGGACGGAGAGGATGAGGAGGGAAAAGAGGATGGGAGAGAAGAAGGCAAAGAG $\begin{array}{llllllllllllllllllll}S & R & V & D & G & E & D & E & E & G & K & E & D & G & R & E & E & G & K & E\end{array}$ GATCCAGAGAAGGGGGCCCTAACCACCCAGGATGAAAATGGCCAGACCAAGCGCAAGTTG $\begin{array}{lllllllllllllllllllll}D & P & E & K & G & A & L & T & T & Q & D & E & N & G & Q & T & K & R & K & L\end{array}$ CAGGCAGGCAAGAAGAGTCAGGACAAGCAGAAAAAGCGGCAGCGAGACCGCATGGTGGAA $\begin{array}{lllllllllllllllllllll}Q & A & G & K & K & S & Q & D & K & Q & K & K & R & Q & R & D & R & M & V & E\end{array}$ AGGATCCAGTTTGTGTGTTCTCTGTGCAAATACCGGACCTTCTATGAGGACGAGATGGCC

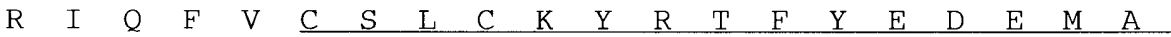
AGCCATCTTGACAGCAAGTTCCACAAGGAACACTTTAAGTACGTAGGCACCAAGCTCCCT $\begin{array}{lllllllllllllllllllllllll}S & H & L & D & S & K & F & H & K & E & H & F & K & Y & V & G & T & K & L & P\end{array}$ AAGCAGACGGCTGACTTTCTGCAGGAGTACGTCACTAACAAGACCAAGAAGACAGAGGAG $\begin{array}{lllllllllllllllllllll}K & Q & T & A & D & F & L & Q & E & Y & V & T & N & K & T & K & K & T & E & E\end{array}$ CTCCGAAAAACCGTGGAGGACCTTGATGGCCTCATCCACCAAATCTACAGAGACCAGGAT $\begin{array}{llllllllllllllllllll}L & R & K & T & V & E & D & L & D & G & L & I & H & Q & I & Y & R & D & Q & D\end{array}$ CTGACCCAGGAAATTGCCATGGAGCATTTTGTGAAGAAGGTGGAGGCAGCCCATTGTGCA $\begin{array}{lllllllllllllllllllll}\mathrm{L} & \mathrm{T} & \mathrm{Q} & \mathrm{E} & \mathrm{I} & \mathrm{A} & \mathrm{M} & \mathrm{E} & \mathrm{H} & \mathrm{F} & \mathrm{V} & \mathrm{K} & \mathrm{K} & \mathrm{V} & \mathrm{E} & \mathrm{A} & \mathrm{A} & \mathrm{H} & \mathrm{C} & \mathrm{A}\end{array}$ GCCTGCGACCTCTTCATTCCCATGCAGTTTGGGATCATCCAGAAGCATCTGAAGACCATG $\begin{array}{llllllllllllllllllll}A & C & D & L & F & I & P & M & Q & F & G & I & I & Q & K & H & L & K & T & M\end{array}$ GATCACAACCGGAACCGCAGGCTCATGATGGAGCAGTCCAAGAAGTCCTCCCTCATGGTG $\begin{array}{llllllllllllllllllllll}\mathrm{D} & \mathrm{H} & & \mathrm{N} & \mathrm{R} & \mathrm{N} & \mathrm{R} & \mathrm{R} & \mathrm{L} & \mathrm{M} & \mathrm{M} & \mathrm{E} & \mathrm{Q} & \mathrm{S} & \mathrm{K} & \mathrm{K} & \mathrm{S} & \mathrm{S} & \mathrm{L} & \mathrm{M} & \mathrm{V}\end{array}$ GCCCGCAGTATTCTCAACAACAAGCTCATCAGCAAGAAGCTGGAGCGCTACCTGAAGGGC $\begin{array}{lllllllllllllllllllll}A & \mathrm{R} & \mathrm{S} & \mathrm{I} & \mathrm{L} & \mathrm{N} & \mathrm{N} & \mathrm{K} & \mathrm{L} & \mathrm{I} & \mathrm{S} & \mathrm{K} & \mathrm{K} & \mathrm{L} & \mathrm{E} & \mathrm{R} & \mathrm{Y} & \mathrm{L} & \mathrm{K} & \mathrm{G}\end{array}$ GAGAACCCTTTCACCGACAGCCCCGAGGAGGAGAAGGAGCAGGAGGAGGCTGAGGGCGGT $\begin{array}{llllllllllllllllllll}E & N & P & F & T & D & S & P & E & E & E & K & E & Q & E & E & A & E & G & G\end{array}$ GCCCTGGACGAGGGGGCGCAGGGCGAAGCGGCAGGGATCTCGGAGGGCGCAGAGGGCGTG $\begin{array}{llllllllllllllllllllllllllll}A & L & D & E & G & A & Q & G & E & A & A & G & I & S & E & G & A & E & G & V\end{array}$
60

120

25

180

45

240

65

300

85

360

105

420

125

480

145

540

165

600

185

660

205

720

225

780

245

840

265

900

285

960

305

1020

325

1080

345

1140

365

1200

385

1260

405

1320

425

1380

445

1440

465

1500

485

1560

505

1620

525

1680

545

1740

565

1800

585 
hAKAP 95 rAKAP 95 NAKAP 95

hАKAP 95

IAKAP 95

NAKAP 95

hAKAP 95 IAKAP 95

NAKAP 95

hAKAP 95

rAKAP 95

NAKAP 95

hАKAP 95 IAKAP 95 NAKAP 95

hAKAP 95 rAKAP 95 NAKAP 95

hAKAP 95

IAKAP 95

NAKAP 95

hAKAP 95

IAKAP 95

NAKAP 95

hAKAP 95 IAKAP 95

NAKAP 95

hАKAP 95 rAKAP 95 NAKAP 95

hAKAP 95 IAKAP 95 NAKAP 95

hAKAP 95 IAKAP 95 NAKAP 95

hAKAP 95 rAKAP 95

b NAKAP 95

CCGGCGCAGCCTCCCGTGCCCCCAGAGCCAGCCCCCGGGGCCGTGTCGCCGCCACCGCCG $\begin{array}{llllllllllllllllllll}P & A & Q & P & P & V & P & P & E & P & A & P & G & A & V & S & P & P & P & P\end{array}$ CCGCCCCCAGAGGAGGAGGAGGAGGGCGCCGTGCCCTTGCTGGGTGGGGCGCTGCAACGC $\begin{array}{llllllllllllllllllllllllllllll}\mathrm{P} & \mathrm{P} & \mathrm{P} & \mathrm{E} & \mathrm{E} & \mathrm{E} & \mathrm{E} & \mathrm{E} & \mathrm{G} & \mathrm{A} & \mathrm{V} & \mathrm{P} & \mathrm{L} & \mathrm{L} & \mathrm{G} & \mathrm{G} & \mathrm{A} & \mathrm{L} & \mathrm{Q} & \mathrm{R}\end{array}$ CAGATCCGCGGCATCCCGGGCCTCGACGTGGAGGACGACGAGGAGGGCGGCGGGGGCGCC $\begin{array}{lllllllllllllllllllll}\mathrm{Q} & \mathrm{I} & \mathrm{R} & \mathrm{G} & \mathrm{I} & \mathrm{P} & \mathrm{G} & \mathrm{L} & \mathrm{D} & \mathrm{V} & \mathrm{E} & \mathrm{D} & \mathrm{D} & \mathrm{E} & \mathrm{E} & \mathrm{G} & \mathrm{G} & \mathrm{G} & \mathrm{G} & \mathrm{A}\end{array}$ CCGTGACccgagctcggggcgggcggagccogcgtggccgaacgtggaaaccaaacct $\underline{\underline{a a}}$ $\mathrm{P} \quad$ *

a taaagttttccatccc

1860

605

1920

625

1980

645

2040

646 2057

MDOGYGGYGAWSAGPANTOGAYGTGVASWOGYENYNYYGAQNTSVTTGATY SYG PASWEA 1 MEOSYGGYGAWSAGPANT OGTYGSGVASWOGYENYSYYNAONTSVPTGTPY SYGPASWEA $1 \sim \sim \sim M S Y T G F V Q G S E T T L Q S T Y S D T S A Q P T C D Y G Y G T W N S G T N R G Y E G Y G Y G Y G Y G Q D N T$

61 AKANDGGLAAGA PAMHMASYGPEPCTDNS... DSLIAK INQRLDMMSKEGGRGGSGGGGE

61 TKASDG GLAA SSAMHVA SFAP E P T TNS. . D D L IAK INQR LDMESKE G G G GISSGGE

57 TNYG YGMATSHSWEMPS SDTNANTSA SGSA SAD SVLSRINQR LDMV . PHLETDMMQG GVY

118 GIQDRES SF RF P PESYDSR P CLPEHNPYR P SY Y DYEF DLGSDRNGSFGGQYSECRDPA 118 GMQDRDSSFR F Q PYESYDSR P MPEHTPYRP SYSYDYDEDLGTDRNG SFGGTFNDCRDPT 116 G. . . . SGGERYDSY E SCD SRAVLSERDLYRSGYDYS . . ELD PEMEMAYEG QYDAYRDQF

178 RERGSLDG FMRGRGOGRFODRSNPGTFMRSDPFVPPAASSEPLSTPWNELNYVGGRGLGG 178 PERGALDG FLRGRG OGRFODRSNSSTFIR D P FMP PSAS S P L TTWSELN YMGGRG LGG 169 RMRG. . NDTF G PRAQGWARDARSGR ........ PMASG . . YGRMWEDPMGARGQCMSG

238 PSP SRPP P LF SQSMAP DYGV. MGMQGAGGYD STMPYGCGR SOPRMRDRDRPKRRGFDRF

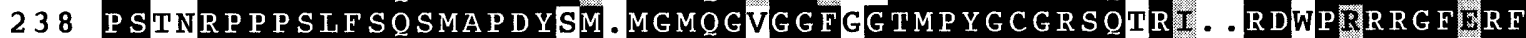
215 ASRL . . . PSLF S ONI IPEY GMF QGMRGGGAFPGG SRFGFGFGNGMKQMRRTWKTWTTADE

297 GP DGTGRKRKOFOLY EEP DTKLARVD.SEG DFSENDDAA.GDFRSG . . . . . . . . . . . 295 G P DNMG RKRKPFPLYEEP DAKLARAD.SEGDLSENDDGA.GDLRSG. . . . . . . . . 272 RTKK. . KKRKQGGSPDEP DSKATRTDCSDNSDSDNDEGTEGEATEGLEGTEAVEKGSRVD

341 DEEFKGEDELCDSGRQRGEK . . . . EDEDEDVKK . . . . RREKQRRRDRTRDRAADR IQF 339 DEEFRGEDDLCD SRKORGEK . . . . EDEDEDVKK . . . . . RREKQRRRDRMRDRAADRIQF 330 GEDEEGKEDGREEGKED PEKGAL T TQDENGQTKRKLQAG KKSQDKQKKRQR DRMVER I O F

391 ACSVCKFRSFDDEEIQKHLQSKFHKETLRF ISTKLPDKTVEFLOEY IVNRNKK I EKRRQF 389 ACSVCKFRSFEDEEIQKHLQSKFHKETLRF ISTKLPDKTVEFLQEY ITNRNKK IEKRRQE 390 VC SIC KYRTFY EDEMASHLDS K F K EH F KYVGTK LPKQTADE LQEYVTNKTKKTEELRKT

451 LMEKETAKPK. . . . PDPFKG I GQEHFFKK I EAAHC LACDML I P A QPOLLQRH LHSVDHNH 449 LLEKESPK PK. . . P D P FKG I GQEHF F KRIEAAHC LAC DML I PAQHQLLQRH LHSVDHNH 450 VEDLDGL IHQI Y RD QDLTQEIAME HFVKKVEAAH CAAC DLFI PMQF G I IQKH LKTMDHNR

507 NRRLAAEQFKKT S LHVAKSVLNNRH IVKMLEKYLKGEDPFTSETVDPEMEGDDNLGGEDK 505 NRRLAAEQFKKT S LHVAKSVLNNKH IVKMLEKY LKG ED P FVNETADLETEG DENLG. . EE 510 NRR LMMEOSKKSS LMVARSTLNNKIISKKL ERY LKG ENP FTDSPEEEKEQEEAEGGALDE

567 KETPEEVAADVLAEVITAAVRAVDGEGAPAPESSG EPAEDEGPTDTAEAGSDPQAEQLLF 563 KETPEEVAAEVLAEVITAAVKAVEGDGEPAAEHSDVLAEVEG PVDTAEAG SDSHTGKLLE 570 GAQGEAAG I SEGAEGVPAQ. P PVPPEPAPGAVSP P PP P... PPEEEEEGAVPLLGGALQ

627 EOVPCGTAHE. KGVPKARSEAAEAgNGAE TMAAEAESAQTRVAPAPAAADAEVEQTDAE 623 EQT. CETASETRNMEDMARG EAAEARNEAAVPAAAAGSPVPVIA. IPG ILEDELEQTDAE 625 RQIRGIPGLDVEDDEEGGGGAP*

685 SKDAVPTE*

681 AKD. TPTE*

Fig. 1. Continued 


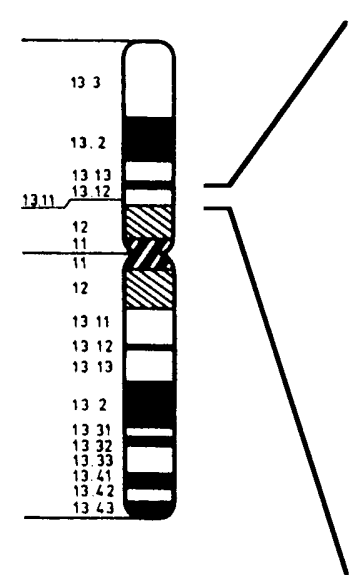

a

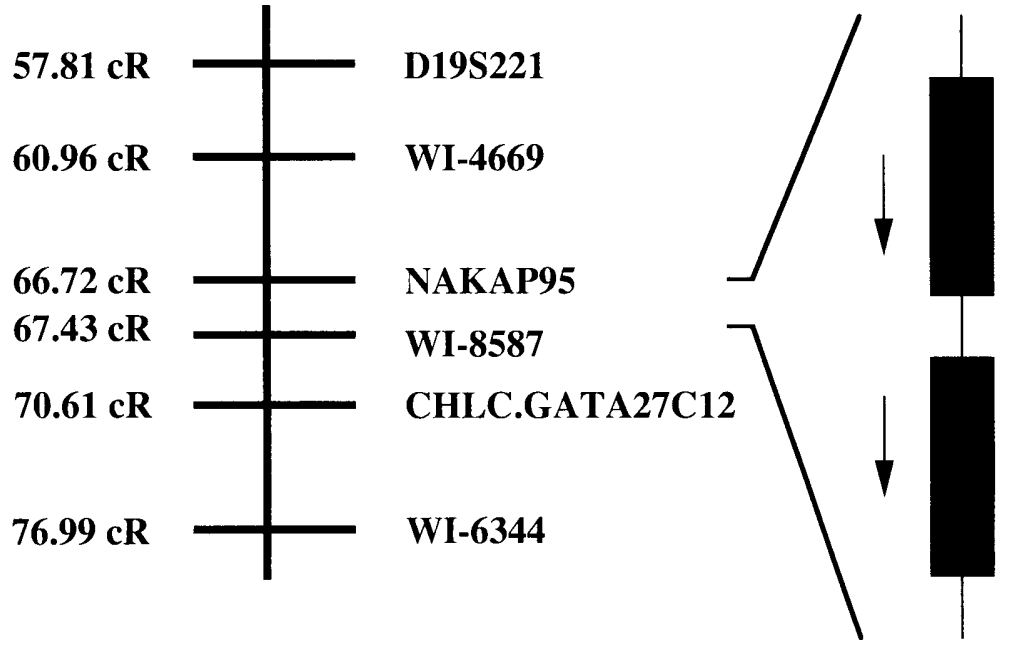

NAKAP95

AKAP95

ATG

NAKAP95

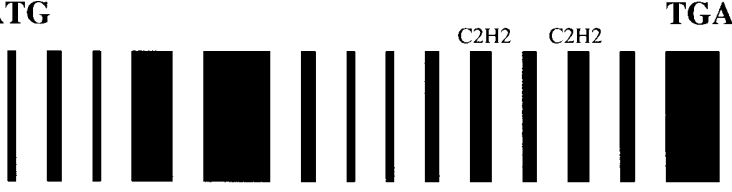

ATG

AKAP95
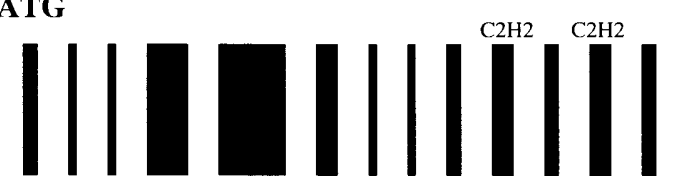

TGA

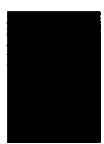

14
Fig. 2. a Chromosomal placement of the NAKAP95 gene at a relative distance to framework markers on the WICGR radiation hybrid map of the human genome (http://carbon.wi.mit.edu:8000/cgi-bin/contig/ phys_map). The approximate corresponding cytogenetic location of the gene on chromosome 19p13.11-p13.12 region is indicated. Distances are in centirays (cR) from the top of the chromosome 19 linkage group. b Schematic exon-intron structure of human NAKAP95 and AKAP95 genes

Table 1. Intron-exon boundaries of the NAKAP95 gene

\begin{tabular}{|c|c|c|c|}
\hline Exon No. & Exon size $^{a}$ & Splice acceptor ${ }^{b}$ & Splice donor ${ }^{b}$ \\
\hline 1 & 58 & & AGCTACACAG gtgggcctggc \\
\hline 2 & 75 & ctttatgtttag GCTTTGTCCA & TGIGATTATG gtaagtgtggac \\
\hline 3 & 33 & tactttttgtag GATATGGAAC & ACAAATAGAG gcaagtgtcatt \\
\hline 4 & 241 & ccctcactgcag GCTACGAGGG & GTGGAGAAAG gtgagtggacac \\
\hline 5 & 454 & atgcctctgcag GTATGACTCT & CGACTTCCGA gtgagtggagge \\
\hline 6 & 97 & cttatgacccag ACCAAGAAGA & TCAGACAATG gtgagcccacta \\
\hline 7 & 71 & tttcccttcag ATGAGGGCAC & CTCCAGAGTG gtaagaggctct \\
\hline 8 & 64 & tgaaattgttag GACGGAGAGG & CCAGAGAAGG gtgagttttcct \\
\hline 9 & 109 & ctgctcccacag GGGCCCTAAC & TGGTGGAAAG gtaaccagcttc \\
\hline 10 & 142 & ttcctgtggcag GATCCAGTTT & стTTCTGCAG gtgagccttgga \\
\hline 11 & 106 & tattccttgcag GAGTACGTCA & CTGACCCAGG gtgaggagattt \\
\hline 12 & 131 & tccccactacag AAATTGCCAT & GAACCGCAGG gtgagtggccac \\
\hline 13 & 96 & ccctgcccgcag CTCATGATGG & CTACCTGAAG gtgaggcactgg \\
\hline 14 & 380 & ccctccccacag CCGCTCTTGG & AAGGGTAGGG \\
\hline
\end{tabular}

Intron-exon junctions were established by comparison of cDNA and genomic sequences

${ }^{a}$ Size in basepairs

${ }^{\mathrm{b}}$ Sequences at the splice junction. Exonic sequences are shown in capital letters, with intronic sequences

shown in lowercase letters. Invariant nucleotides (ag/gt, gc) are in boldface type 


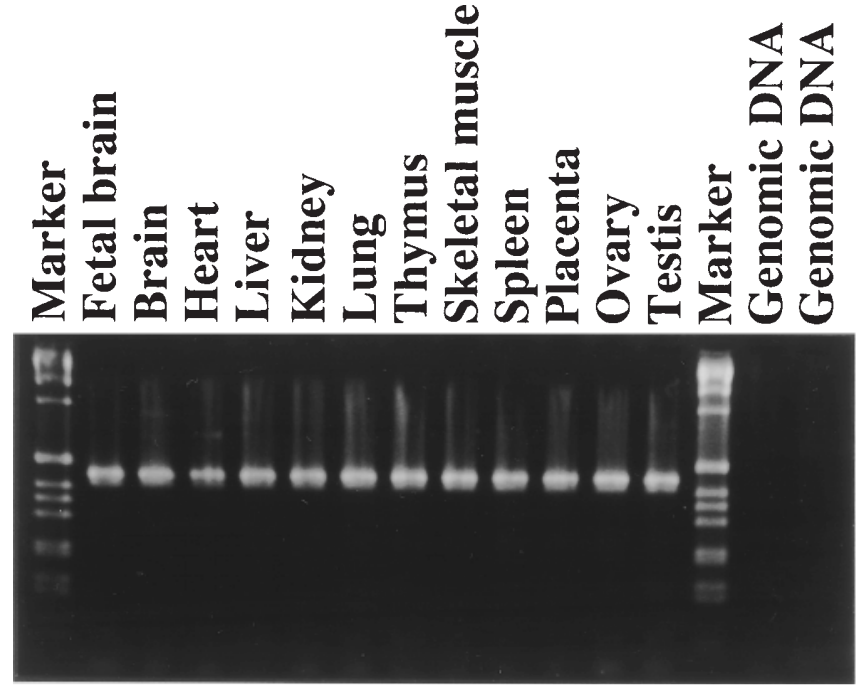

Fig. 3. Tissue distribution analysis, using reverse transcription coupled-polymerase chain reaction (RT-PCR). The 12 tissues and genomic DNA examined are indicated above each lane. The templates of the human tissues of poly $(\mathrm{A})+$ RNAs were purchased from Clontech (Palo Alto, CA, USA). The cDNA templates for RT-PCR were synthesized from $2 \mu \mathrm{g}$ of poly $(\mathrm{A})+$, using excess amounts of Superscript II reverse transcriptase (GIBCO BRL, Gaithersburg, MD, USA) and random hexamer primers. PCR was carried out in a final volume of $10 \mu \mathrm{l}$ containing $1 \times$ LA-PCR buffer (Takara, Kyoto, Japan), $2 \mu \mathrm{M}$ each primer, $200 \mu \mathrm{M}$ each dNTP, $1 \mu \mathrm{l}$ of template DNA, and 0.01 units of LA-Taq DNA polymerase (Takara). Temperatures and time schedule were: 30 cycles of $95^{\circ} \mathrm{C}$ for $20 \mathrm{~s}$ and $62^{\circ} \mathrm{C}$ for $1 \mathrm{~min}$. PCR products were separated on $2.5 \%$ Nusieve GTG agarose gel (FMC, Rockland, ME, USA) with a 1-kb ladder DNA marker (GIBCO BRL)

The exon-intron boundaries of the human NAKAP95 and $A K A P 95$ genes were determined by aligning the cDNA sequence with the genomic sequence from the two cosmid clones (accession numbers, AC005785, AC006128) (Fig. 2b). As shown in Table 1, all but one of the splicing sites conformed to the canonical splicing acceptor and donor rule of AG-GT; one was AG-GC. The NAKAP95 gene was divided into 14 exons, which ranged in size from $33 \mathrm{bp}$ (exon 3) to $454 \mathrm{bp}$ (exon 5). Exons 1 and 14 contained the ATG and TAG codons, respectively. The exon-intron boundary of the human $A K A P 95$ gene was also determined in the same manner, revealing that human NAKAP95 and $A K A P 95$ had a very similar gene structure (Fig. 2b). In both cases, the $\mathrm{C} 2 \mathrm{H} 2$ type zinc finger motif resided in exons 10 and 12 . These results strongly argue that the NAKAP95 and AKAP95 genes could have been established by tandem gene duplication.

\section{References}

Boynton AL, Whitfield JF (1983) The role of cAMP in cell proliferration: a critical assessment of the evidence. Adv Cyclic Nucleotide Res 15:193-294

Carr DW, Stofko-Hahn RE, Fraser ID, Cone RD, Scott JD (1992) Localization of the cAMP-dependent protein kinase to the postsynaptic densities by A-kinase anchoring proteins. Characterization of AKAP 79. J Biol Chem 267:16816-16823

Chen Q, Lin RY, Rubin CS (1997) Organelle-specific targeting of protein kinase AII (PKAII). Molecular and in situ characterization of murine A kinase anchor proteins that recruit regulatory subunits of PKAII to the cytoplasmic surface of mitochondria. J Biol Chem 272:15247-15257

Coghlan VM, Langeberg LK, Fernandez A, Lamb NJ, Scott JD (1994) Cloning and characterization of AKAP 95, a nuclear protein that associates with the regulatory subunit of type II cAMP-dependent protein kinase. J Biol Chem 269:7658-7665

Edelman AM, Blumenthal DK, Krebs EG (1987) Protein serine/threonine kinases. Annu Rev Biochem 56:567-613

Eide T, Coghlan V, Orstavik S, Holsve C, Solberg R, Skalhegg BS, Lamb NJ, Langeberg L, Fernandez A, Scott JD, Jahnsen T, Tasken K (1998) Molecular cloning, chromosomal localization, and cell cycle-dependent subcellular distribution of the A-kinase anchoring protein, AKAP95 Exp Cell Res 238:305-316

Faux MC, Scott JD (1996) Molecular glue: kinase anchoring and scaffold proteins. Cell 85:9-12

Hausken ZE, Dell'Acqua ML, Coghlan VM, Scott JD (1996) Mutational analysis of the A-kinase anchoring protein (AKAP)-binding site on RII. Classification of side chain determinants for anchoring and isoform selective association with AKAPs. J Biol Chem 271:29016-29022

Hausken ZE, Scott JD (1996) Properties of A-kinase anchoring proteins. Biochem Soc Trans 24:986-991

Keryer G, Rios RM, Landmark BF, Skalhegg B, Lohmann SM, Bornens M (1993) A high-affinity binding protein for the regulatory subunit of cAMP-dependent protein kinase II in the centrosome of human cells. Exp Cell Res 204:230-240

Kozak M (1987) At least six nucleotides preceding the AUG initiatior codon enhance translation in mammalian cells. J Mol Biol 196:947950

Krebs EG, Beavo JA (1979) Phosphorylation-dephosphorylation of enzymes. Annu Rev Biochem 48:923-959

Lester LB, Coghlan VM, Nauert B, Scott JD (1996) Cloning and characterization of a novel A-kinase anchoring protein. AKAP 220, association with testicular peroxisomes. J Biol Chem 271:94609465

Li Y, Ndubuka C, Rubin CS (1996) A kinase anchor protein 75 targets regulatory (RII) subunits of cAMP-dependent protein kinase II to the cortical actin cytoskeleton in non-neuronal cells. J Biol Chem 271:16862-16869

McKnight GS (1991) Cyclic AMP second messenger systems. Curr Opin Cell Biol 3:213-217

McCartney S, Little BM, Langeberg LK, Scott JD (1995) Cloning and characterization of A-kinase anchor protein 100 (AKAP100). A protein that targets A-kinase to the sarcoplasmic reticulum. J Biol Chem 270:9327-9333

Nauert JB, Klauck TM, Langeberg LK, Scott JD (1997) Gravin, an autoantigen recognized by serum from myasthenia gravis patients, is a kinase scaffold protein. Curr Biol 7:52-62

Roesler WJ, Vandenbark GR, Hanson RW (1988) Cyclic AMP and the induction of eukaryotic gene transcripton. J Biol Chem 263:90639066

Rubin CS (1994) A kinase anchor proteins and the intracellular targeting of signals carried by cyclic AMP. Biochim Biophys Acta 1224:467-479

Saito T, Seki N, Ishii H, Ohira M, Hayashi A, Kozuma S, Hori T (1997) Complementary DNA cloning and chromosomal mapping of a novel phosphatidylinositol kinase gene. DNA Res 4:301-305

Schmidt PH, Dransfield DT, Claudio JO, Hawley RG, Trotter KW, Milgram SL, Goldenring JR (1999) AKAP350, a multiply spliced protein kinase A-anchoring protein associated with centrosome. J Biol Chem 274:3055-3066

Scott JD, Macartney S (1994) Localization of A-kinase through anchoring proteins. Mol Endocrinol 8:5-11

Seki N, Nimura Y, Ohira M, Saito T, Ichimiya S, Nomura N, Nakagawara A (1997) Identification and chromosome assignment of a human gene encoding a novel phosphatidylinositol-3 kinase. DNA Res 4:355-358

Seki N, Muramatsu M, Sugano S, Suzuki Y, Nakagawara A, Ohhira M, Hayashi A, Hori T, Saito T (1998) Cloning, expression analysis, and chromosomal localization of HIP1R, an isolog of huntingtin interacting protein (HIP1). J Hum Genet 43:268-271 
Seki N, Hattori A, Hayashi A, Kozuma S, Ohira M, Hori T, Saito T (1999) Structure, expression profile and chromosomal location of an isolog of DNA-PKcs interacting protein (KIP) gene. Biochim Biophys Acta 1444:143-147

Taylor SS, Buechler JA, Yonemoto W (1990) cAMP-dependent protein kinase: framework for a diverse family of regulatory enzymes. Annu Rev Biochem 59:971-1005

Ueki N, Oda T, Kondo M, Yano K, Noguchi T, Muramatsu M (1998) Selection system for genes encoding nuclear-targeted proteins. Nat Biotechnol 16:1338-1342

Ueki N, Seki N, Yano K, Ohira M, Saito T, Masuho Y, Muramatsu M (1999a) Isolation and chromosomal assignment of human genes encoding cofactor of LIM homeodomain proteins, CLIM1 and CLIM2. J Hum Genet 44:112-115

Ueki N, Seki N, Yano K, Saito T, Masuho Y, Muramatsu M (1999b) Isolation and chromosomal assignment of a human gene encoding protein inhibitor of activated STAT3 (PIAS3). J Hum Genet 44:193196

Ueki N, Seki N, Yano K, Saito T, Masuho Y, Muramatsu M (1999c) Isolation and characterization of a novel human gene (HFB30) which encodes a protein with a RING finger motif. Biochim Biophys Acta 1445:232-236 